\section{Ultra Staging to Unmask the Prescribing of Adjuvant Therapy in Cancer Patients: The Future Opportunity to Image Micrometastases Using Total-Body ${ }^{18}$ F-FDG PET Scanning}

TO THE EDITOR: The unrivaled sensitivity of PET for imaging molecular interactions remains largely unexploited in oncology. At present, PET mainly uses ${ }^{18}$ F-labeled FDG PET to diagnose and stage cancer by imaging primary and macroscopic metastatic deposits. We wish to draw attention to the opportunity of extending the use of ${ }^{18}$ F-FDG PET to detect and quantify micrometastatic disease. The methodology we propose - using a PET scanner that covers the entire length of the patient and optimized tumor-tobackground tissue contrast-will maximize the sensitivity of ${ }^{18} \mathrm{~F}$ FDG PET, offering the best chance for detection and quantification of micrometastases.

For cancer patients in the adjuvant setting, clinical decisions after radical surgical treatment of the primary tumor are based on risk factors identified from the surgically removed primary tumor and regional nodes. Patients considered at risk of disease relapse are presumed to have micrometastatic disease occult to current detection methods: all are therefore treated with toxic adjuvant chemotherapy. Until imaging and staging methods are advanced enough to provide evidence of micrometastatic status, we are restricted to this masked treatment approach. Although this approach improves overall relapse and survival rates, the disadvantages are: first, risk overestimation leading to overtreatment; second, inability to define patients who do or do not benefit; third, inability to assess real-time treatment response; and fourth, treatment toxicity, especially long-term side effects. For example, in the United States over 140,000 patients are diagnosed each year with colorectal cancer. On the basis of indicators of statistical risk, approximately $27 \%$ of early-stage (I + II) and $61 \%$ of late-stage (III + IV) colorectal cancer patients receive chemotherapy in addition to surgery (1). Yet cure is likely in a large number without adjuvant treatment, as in stage III node-positive colon cancer, for which $44 \%$ of patients were reported disease-free 5 y after surgery alone (2). Furthermore, for those who experience the inconvenience and toxicity of adjuvant treatment, not until months later does it become apparent that many patients may not have benefited (3). A method to detect and quantify micrometastasis would therefore greatly affect the staging and management of patients in the adjuvant setting, supporting the rational assessment of adjuvant chemotherapy risk-benefit for individual patients and sparing unnecessary treatment morbidity and costs. Such a method could also be used to optimize patient response to chemotherapeutics on a personalized medicine basis and would additionally support the assessment of new anticancer drugs to accelerate their clinical development.

The clinical usefulness of ${ }^{18} \mathrm{~F}-\mathrm{FDG}$ PET for macroscopic tumor detection has been restricted in oncology as a result of the limited sensitivity and specificity of the methodologies used (4). It is possible that MR imaging may supersede ${ }^{18} \mathrm{~F}$-FDG PET for gross

COPYRIGHT (c) 2014 by the Society of Nuclear Medicine and Molecular Imaging, Inc. tumor localization, given its higher spatial resolution and radiation-free capabilities for measuring tumor glucose concentrations (5). However, the intrinsically higher sensitivity of PET for detecting low levels of tracer makes ${ }^{18} \mathrm{~F}-\mathrm{FDG}$ PET better placed to detect microscopic disease. Nevertheless, improvements in sensitivity and specificity, and detection throughout the body, would be necessary. This need could be addressed in 2 ways. First, the contrast between tumor and background tissue may be increased by approximately 4-fold if the interval between administration of ${ }^{18} \mathrm{~F}-\mathrm{FDG}$ and patient scanning is increased from the routinely used $60 \mathrm{~min}$ to about $300 \mathrm{~min}$ : this increase in the interval results in wider distribution of tracer, greater accumulation in tumor, extended renal excretion, and a higher washout from normal tissue $(6,7)$. Second, the development of total-body PET-a 2-m-long tunnel of coincidence detectors operating in the time-of-flight mode (Fig. 1) - could significantly increase the efficiency of tracer detection compared with conventional PET scanning at equivalent injected doses $(8,9)$. We propose an imaging protocol that would involve administering about $740 \mathrm{MBq}(20 \mathrm{mCi})$ of ${ }^{18} \mathrm{~F}$-FDG to a patient with an interval of 3 half-lives ( $\sim 330 \mathrm{~min})$ before a totalbody PET scan. Here it is predicted that the improvement in the quality of the image data would be equivalent to a 100 -fold gain in noise-equivalent counts compared with using a conventional scanner and imaging at $60 \mathrm{~min}$ after tracer administration. This improvement results from the expected increase in contrast (6) combined with the improved detection efficiency of the total-body scanner $(8,9)$ and accounts for the decay of the radiotracer during the extended uptake time. Therefore, we hypothesize that wholebody localization and quantification of microscopic disease should be possible-either as detectable hot spots or as raised mean levels of tissue and organ concentrations. Enhanced whole-body detection of highly differentiated tumor would be achieved, although assessment of global activity in common sites of metastasis, such as lymph basins, may be needed to ensure detection of early disease.

Challenges in quantifying tissue concentrations are expected using this paradigm but are considered surmountable-for example, resolution or contrast losses due to respiratory motion, and compensation of blood tracer activity remaining within the vasculature. To assess potential early microscopic disease presenting as raised mean tissue activity levels, time series scans and kinetic modeling methods may also need to be used and refined.

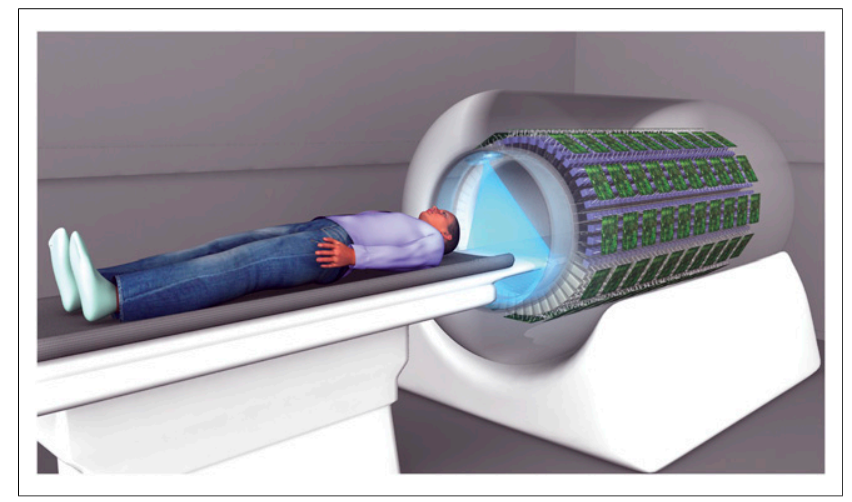

FIGURE 1. Schematic representation of whole-body PET machine (EXPLORER), as previously described and simulated $(8,9)$. 
The additional benefits of using kinetic analysis of serial ${ }^{18} \mathrm{~F}-\mathrm{FDG}$ PET scans would be a gain in tumor glycolytic metabolism information relating to tumor biology and differentiation (e.g., by analyzing slope of ${ }^{18}$ F-FDG uptake over time), which could valuably increase the utility of the method for ultra staging, and thus patient management and adjuvant treatment decisions.

This proposed total-body PET-based imaging paradigm could offer the possibility of dramatically extending the proven sensitivity of ${ }^{18} \mathrm{~F}$-FDG for tumor detection, enabling for the first time the geographic distribution and quantification of microscopic cancer throughout the body. This paradigm also offers the opportunity to obtain information to characterize tumor grade. Thus, ${ }^{18} \mathrm{~F}$-FDG PET has the potential to become an efficient assessment tool for ultra staging, which could have a significant impact on primary and adjuvant therapy decisions, particularly if developed and used in combination with complementary assays of circulating tumor cells (10). In the adjuvant setting, this tool could meet the need for improved patient selection for, and individualized tailoring of, adjuvant therapy, providing significant patient and economic benefit.

\section{REFERENCES}

1. Siegel R, DeSantis C, Virgo K, et al. Cancer treatment and survivorship statistics, 2012. CA Cancer J Clin. 2012;62:220-241.

2. Moertel CG, Fleming TR, Macdonald JS, et al. Fluorouracil plus levamisole as effective adjuvant therapy after resection of stage III colon carcinoma: a final report. Ann Intern Med. 1995;122:321-326.

3. Saif MW. The potential use of targeted agents for the treatment of colorectal cancer in the adjuvant setting. Medscape Oncology website. http://www.medscape.org/ viewarticle/521902. Published 2006. Accessed February 12, 2014.

4. Fletcher JW, Djulbegovic B, Soares HP, et al. Recommendations on the use of ${ }^{18}$ F-FDG PET in oncology. J Nucl Med. 2008;49:480-508.
5. Walker-Samuel S, Ramasawmy R, Torrealdea F, et al. In vivo imaging of glucose uptake and metabolism in tumors. Nat Med. 2013;19:1067-1072.

6. Basu S, Kung J, Houseni M, Zhuang H, Tidmarsh GF, Alavi A. Temporal profile of fluorodeoxyglucose uptake in malignant lesions and normal organs over extended time periods in patients with lung carcinoma: implications for its utilization in assessing malignant lesions. Q J Nucl Med Mol Imaging. 2009;53:9-19.

7. Cheng G, Alavi A, Lim E, Werner TJ, Del Bello CV, Akers SR. Dynamic changes of FDG uptake and clearance in normal tissues. Mol Imaging Biol. 2013;15:345-352.

8. Badawi RD, Poon JK, Surti S, et al. EXPLORER, an ultrasensitive total-body PET scanner: application feasibility simulations. Presented at the World Molecular Imaging Congress on September 19, 2013. World Molecular Imaging Society website. http://www.wmis.org/abstracts/2013/data/papers/LBAP125.htm. Accessed February 12, 2014

9. Poon JK, Dahlbom ML, Moses WW, et al. Optimal whole-body PET scanner configurations for different volumes of LSO scintillator: a simulation study. Phys Med Biol. 2012;57:4077-4094.

10. Allen JE, El-Deiry WS. Circulating tumor cells and colorectal cancer. Curr Colorectal Cancer Rep. 2010;6:212-220.

Patricia M. Price*

Ramsey D. Badawi

Simon R. Cherry

Terry Jones

*Imperial College London

B Block, Hammersmith Campus

Du Cane Rd.

London, U.K. W12 ONN

E-mail:p.price@imperial.ac.uk

Published online Mar. 6, 2014.

DOI: 10.2967/jnumed.113.133892 